\title{
AIMI Antonio, con la collaborazione di Raphael TUNESI, Maya e Aztechi
}

\section{Eric Taladoire}

\section{OpenEdition Journals}

Édition électronique

URL : https://journals.openedition.org/jsa/10043

DOI : $10.4000 /$ jsa. 10043

ISSN : 1957-7842

Éditeur

Société des américanistes

\section{Édition imprimée}

Date de publication : 15 juillet 2008

Pagination : 287-289

ISSN : 0037-9174

\section{Référence électronique}

Eric Taladoire, « AIMı Antonio, con la collaborazione di Raphael tunesı, Maya e Aztechi », Journal de la Société des américanistes [En ligne], 94-1 | 2008, mis en ligne le 25 juillet 2008, consulté le 04 septembre 2022. URL : http://journals.openedition.org/jsa/10043; DOl : https://doi.org/10.4000/jsa. 10043

Ce document a été généré automatiquement le 4 septembre 2022.

Tous droits réservés 


\title{
AIMI Antonio, con la collaborazione di Raphael TUNESI, Maya e Aztechi
}

\author{
Eric Taladoire
}

\section{RÉFÉRENCE}

AIMI Antonio, con la collaborazione di Raphael TUNESI, Maya e Aztechi, Mondadori Electa, coll. « Dizionari delle Civiltà » 8, Milano, 2008, 384 p., réf. dissém., ill.

1 Les éditions Mondadori Electa ont récemment lancé une collection originale de Dictionnaires des Civilisations antiques, dont déjà sept volumes sont publiés. Celui sur les Mayas et Aztèques (Maya e Aztechi) est le huitième. Signalons immédiatement que le titre est trompeur, puisque l'ouvrage couvre plus exactement la Mésoamérique, du moins dans son acception traditionnelle. Nous y reviendrons.

Sans pouvoir déterminer si le découpage correspond à un impératif éditorial commun à l'ensemble de la série, le volume se présente sous la forme de cinq grandes sections : les personnages; le pouvoir, les rituels et la vie politique ; la vie quotidienne ; les déités et la religion; les cités. Chaque section compte entre 15 et 25 entrées, dont chacune couvre de deux à six pages. Ce format ne permet évidemment pas à l'auteur, Antonio Aimi, de disposer d'une grande liberté de manœuvre, puisqu'il lui a fallu effectuer des choix difficiles, accepter de ne pas parler de nombreux sites - voire de régions entières comme le Centre-Nord du Mexique ou l'Occident, sans parler du Nord (La Quemada, Casas Grandes) - et laisser de côté des thèmes ou des enjeux, comme les techniques agricoles, les chinampas, par exemple. De même, on peut déplorer la part réduite consacrée aux écritures et aux systèmes calendaires, qui ne font l'objet que de deux rubriques, alors qu'il s'agit d'une manifestation culturelle fondamentale pour le monde mésoaméricain. On peut donc, d'emblée, émettre une réserve, ou plus précisément avertir le lecteur : il ne s'agit que d'un dictionnaire, et non d'un manuel ou d'un ouvrage de synthèse. C'est un outil de divulgation, voire un ouvrage à emporter lors d'un voyage, pour compléter une découverte ou préciser certaines notions. 
3 Cette réserve émise, ce dictionnaire se révèle riche et de très bonne qualité. Chaque rubrique comprend un texte court et synthétique, mais précis et, dans la plupart des cas, au fait des recherches récentes (comme pour ce qui touche à Tenochtitlan et aux fouilles du Templo Mayor). De brefs encarts complètent et enrichissent le texte, en renvoyant le lecteur aux fouilles, à des thèmes complémentaires ou à des références bibliographiques. Mais, surtout, chaque entrée comprend entre deux et une dizaine d'illustrations d'excellente qualité (même si certaines sont parfois trop réduites, à la limite de la lisibilité, comme la carte de la page 371), accompagnées de légendes et de commentaires qui compensent largement les limitations de la partie rédigée. Et c'est dans ce domaine iconographique que l'auteur est parvenu avec brio à dépasser les contraintes du format imposé. Les illustrations comprennent en effet des pièces de l'ensemble des civilisations mésoaméricaines, Occident inclus, et font largement appel aux documents pictographiques ainsi qu'à la multiplicité des techniques. Cela corrige nombre de lacunes et offre à l'utilisateur une vision plus ample et plus complète de l'aire mésoaméricaine.

4 Aimi est ainsi parvenu à donner une vision globale de l'ensemble des civilisations qui se sont succédé sur ce territoire au fil de quelque trois millénaires, ce qui pour un ouvrage de ce type constitue une réussite. On ne peut que regretter, face à ce remarquable travail, un certain nombre d'erreurs ou de lacunes, souvent mineures, mais qui auraient été faciles à éviter. La plus lourde de conséquences est d'ordre chronologique. Dans la section des cités, par exemple, la présentation obéit à une logique géographique, voire «touristique », en commençant par les sites du Haut Plateau central mexicain, pour se poursuivre par les autres sites de Oaxaca ou des environs de Mexico, les sites olmèques et s'achever sur les cités mayas. Le lecteur peu familier risque de s'y perdre car on décrit d'abord des sites du Postclassique (Tula ou Tenochtitlan), pour en venir ensuite au Préclassique (La Venta), puis au Classique (Monte Alban, El Tajín) avant de revenir au Postclassique (Chichen Itza, Tulum), puis à nouveau au Classique (Copán, Tikal, Palenque). Il eût été facile, et infiniment préférable, de présenter les sites en fonction de leur datation, ce qui n'aurait en rien nui à la fonction de dictionnaire, mais aurait permis au lecteur de mieux saisir toute la complexité et la richesse de l'évolution culturelle. À un degré mineur, le même défaut se retrouve dans les autres entrées, même si l'auteur a pris soin de préciser autant que faire se peut dates et provenances. Dans un autre ordre d'idées, on peut noter, de-ci, de-là, de petites erreurs de bibliographie (par exemple, pour les fouilles de Toniná, p. 341, attribuées seulement à Baudez, ou l'absence de renvoi aux travaux récents de Winter pour Monte Alban) et, surtout, s'agissant justement de ces renvois aux fouilles, l'absence des références pertinentes en bibliographie ou en notes. Le lecteur est appâté, mais laissé à lui-même. Certes, le format de l'ouvrage ne permet pas la présence d'une telle bibliographie mais, dans ces conditions, pourquoi inclure ces renvois aux fouilles?

Il serait loisible de mentionner d'autres inexactitudes et de donner ici l'impression d'une appréciation négative, que ce volume ne mérite en rien. Il était indispensable d'attirer l'attention sur les quelques erreurs, plus imputables au concept même de la collection qu'à l'auteur, pour souligner l'intérêt et la qualité de ce dictionnaire. Il ne peut, ni ne veut, être un manuel, mais un guide, une aide pour appréhender des civilisations encore trop mal connues, et il y parvient au-delà de ce que l'on peut l'espérer, car c'est aussi un livre d'art, maniable, accessible et à un prix abordable. Que demander de plus? 


\section{AUTEURS}

ERIC TALADOIRE

Université Paris I 